\title{
Stable Fabrication of Zwitterionic Coating Based on Copper-Phenolic Networks on Contact Lens with Improved Surface Wettability and Broad-Spectrum Antimicrobial Activity
}

Gongyan Liu, ${ }^{\dagger}$ Kaijun Li, ${ }^{\dagger}$ Haibo Wang, ${ }^{\perp} \mathrm{Li} \mathrm{Ma},{ }^{\dagger}$ Ling $\mathrm{Yu}^{*} \mathbb{C}$ and $\mathrm{Yu} \mathrm{Nie}^{\S}$

$\dagger$ The Key Laboratory of Leather Chemistry and Engineering of Ministry of Education, College of Biomass Science and Engineering, Sichuan University, Chengdu 610065, China

${ }^{\perp}$ Textile Institute, College of Biomass Science and Engineering, Sichuan University, Chengdu 610065, China

${ }^{\mathbb{C}}$ Department of Ophthalmology, The Affiliated Hospital of Southwest Medical University, Luzhou 646000, China.

§ational Engineering Research Center for Biomaterials, Sichuan University, Chengdu 610064, China

Corresponding Author

*E-mail: oculistlingyu@hotmail.com (Y. L.) 


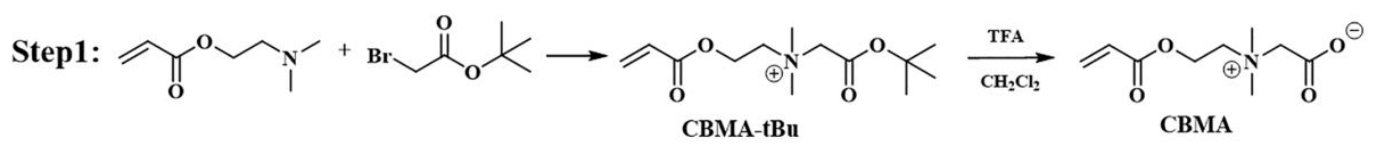

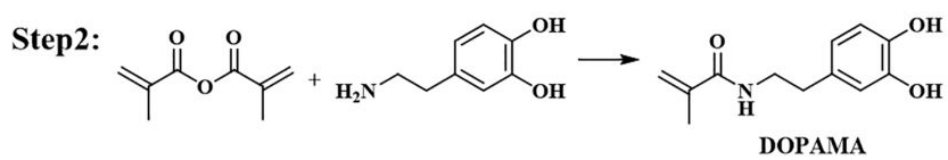

Step3:
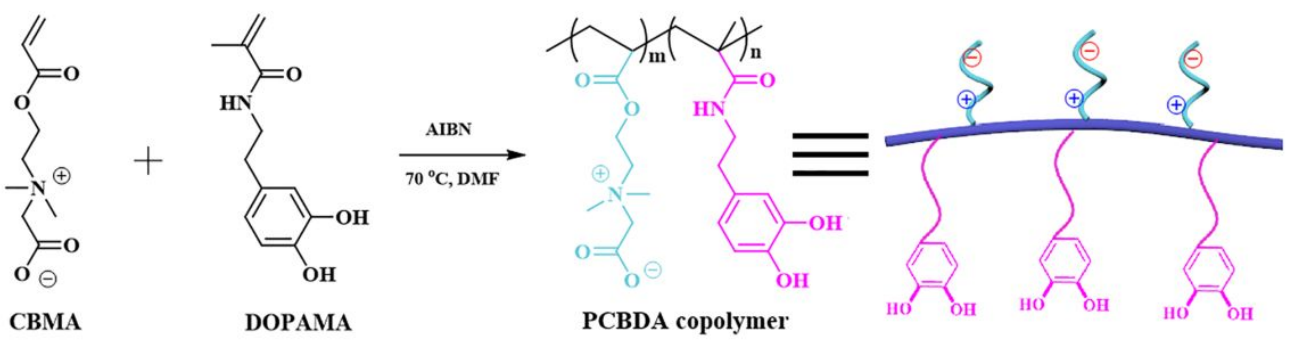

Figure S1. Synthesis route of zwitterionic PCBDA copolymer. 